\title{
CONTAMINACIÓN POR METALES PESADOS EN SUELO PROVOCADA POR LA INDUSTRIA MINERA
}

\section{HEAVY METALS POLLUTION IN SOILS DAMAGED BY MINING INDUSTRY}

\author{
Soraya Puga ${ }^{1}$, Manuel Sosa ${ }^{2}$, Toutcha Lebgue ${ }^{2}$, Cesar Quintana ${ }^{1}$ y Alfredo Campos ${ }^{1}$
}

\section{Resumen}

La extracción minera en el área data del año 1600, provocando disturbios al medioambiente y generando suelos con limitaciones físicas, químicas y biológicas para el establecimiento de vegetación y riesgos a la salud. El objetivo del estudio fue evaluar la contaminación en el suelo por Plomo, Cadmio, Cinc y Arsénico a diferentes distancias y niveles de profundidad y determinar su relación con características físico-químicas. El área se encuentra en San Francisco del Oro, Chih, en el área de influencia de la presa de jales la cual es un área de confinamiento de los desechos de la industria minera, abarcó $3 \mathrm{~km}$ lineales en dirección de vientos dominantes a partir de la fuente de contaminación. Se extrajeron 30 muestras de suelo para análisis de concentraciones de metales y de características físico-químicas a tres diferentes profundidades: 0-40, 40-60 y 60-80 cm en 10 sitios la distancia entre sitios fue de $300 \mathrm{~m}$. La concentración de metales se evaluó utilizando la técnica ICP, las muestras con pequeñas concentraciones se leyeron mediante absorción atómica. El análisis estadístico fue modelos de regresión entre características físico-químicas, distancias, profundidades y concentraciones de metales pesados. Los resultados muestran que la mayor concentración se da en sitios cercanos a los jales, disminuyendo la concentración a medida que se aleja de ellos. Todos los elementos sobrepasan los rangos establecidos por las agencias internacionales. El Arsénico se asoció con distancia a la fuente, textura y materia orgánica; el Plomo con la distancia a la fuente y textura; el Cinc con distancia a la fuente y el Cadmio con la distancia a la fuente, $\mathrm{pH}$ y profundidad de suelo.

Palabras clave: contaminación suelo, minas, presa de jales, metales pesados

\begin{abstract}
Mining in the study area has been carried out for several centuries, causing environmental alterations, generating poor soils for plant establishment and health hazards for humans. The objective was to evaluate presence of Lead, Cadmium, Zinc and Arsenic in soil with variables: distance to the pollution source and soil depth, hence determining relations with soil physicalchemical characteristics. The study area is located south of Chihuahua State. Sampling sites were placed in the area of the mine tailing. These sites covered a lineal area of $3 \mathrm{~km}$ following downward directions to the dominant winds beginning from the pollution source, and taken every $300 \mathrm{~m}$. Ten sites provided thirty soil samples for the analysis of metal concentrations, and for physical-chemical characteristics, from three different soil depths: 0-40, 40-60 and 60-80 cm. Metal concentrations were evaluated through ICP technique. Samples containing small concentrations were read with atomic absorption. A "Regression Analysis Model” was used. The results obtained show that the highest element concentrations were for the sites closest to the tailing deposits, and decreasing with the distances away from the main source. The two sites closest to tailing deposits had higher metal levels in lower soil layers than at the soil surface except for Cadmium. Concentration limits of these metals are higher than any limits recommended by international agencies. Distance to tailing deposits associated with the concentration of all elements. Arsenic correlated with soil texture and organic matter, Lead with texture, Zinc with distance and Cadmium with soil $\mathrm{pH}$ and depth.
\end{abstract}

Key words: soil pollution, mines, tailings, heavy metals

\section{Introducción}

El suelo es alterado como resultado de las actividades mineras. Una de las anomalías biogeoquímicas que se generan al momento de la extracción, es el aumento de la cantidad de microelementos en el suelo convirtiéndolos a niveles de macroelementos los cuales afectan negativamente la biota y calidad de suelo; estos afectan el número, diversidad y actividad de los organismos del suelo, inhibiendo la descomposición de la materia orgánica del suelo (Wong, 2003). Salomons (1995) comenta que los jales son tóxicos para los organismos vivos y son inhibidores de factores ecológicos afectando el crecimiento de las plantas. 
Los suelos que quedan tras una explotación minera contienen todo tipo de materiales residuales, escombros estériles, entre otros, lo que representa graves problemas para el desarrollo de la cubierta vegetal, siendo sus características más notables las siguientes: clase textural desequilibrada, ausencia o baja presencia de la estructura edáfica, propiedades químicas anómalas, disminución o desequilibrio en el contenido de nutrientes fundamentales, ruptura de los ciclos biogeoquímicos, baja profundidad efectiva, dificultad de enraizamiento, baja capacidad de cambio, baja retención de agua y presencia de compuestos tóxicos (García \& Dorronsoro, 2002).

Las características del suelo juegan un papel importante en reducir o aumentar la toxicidad de los metales en el suelo Colombo et al. (1998) comentan que la distribución de los metales pesados en los perfiles del suelo, así como su disponibilidad está controlada por parámetros como propiedades intrínsecas del metal y características de los suelos.

Los metales tienden a acumularse en la superficie del suelo quedando accesibles al consumo de las raíces de los cultivos (Baird, 1999). Las plantas cultivadas en suelos contaminados absorben en general más oligoelementos y la concentración de éstos en los tejidos vegetales está a menudo directamente relacionada con su abundancia en los suelos, y especialmente en la solución húmeda (Kabata-Pendias \& Pendias, 2001) Gulson et al. (1996) mencionan que excesivas concentraciones de metales en el suelo podrían impactar la calidad de los alimentos, la seguridad de la producción de cultivos y la salud del medio ambiente, ya que estos se mueven a través de la cadena alimenticia vía consumo de plantas por animales y estos a su vez por humanos.

Los metales acumulados en la superficie del suelo se reducen lentamente mediante la lixiviación, el consumo por las plantas, la erosión y la deflación. El objetivo del estudio fue evaluar las concentraciones de Plomo (Pb), Cinc (Zn), Cadmio (Cd) y Arsénico (As) en diferentes profundidades de suelo afectado por presas de jales.

\section{Materiales y métodos}

El área de estudio se encuentra en la parte sur del estado de Chihuahua, México en el municipio de San Francisco del Oro, donde se localiza una industria minera la cual cuenta con presas de jales que cubren una superficie de aproximadamente 140 ha (Figura 1). Las coordenadas geográficas del área son 2652 de L.S. y 10551 de L.O. La altitud promedio del área es de 2140 msnm. El tipo de vegetación está constituida por la asociación de pastizal amacollado con bosque de encino y táscate, con matorral espinoso en las partes bajas. El clima esta catalogado como templado sub-húmedo con lluvias en verano, una temperatura media de 16C y una precipitación media de $500 \mathrm{~mm}$. El tipo de suelo tiene una textura arena-arcillo-limoso, un $\mathrm{pH}$ ligeramente ácido y un contenido de materia orgánica de $0.91 \%$.

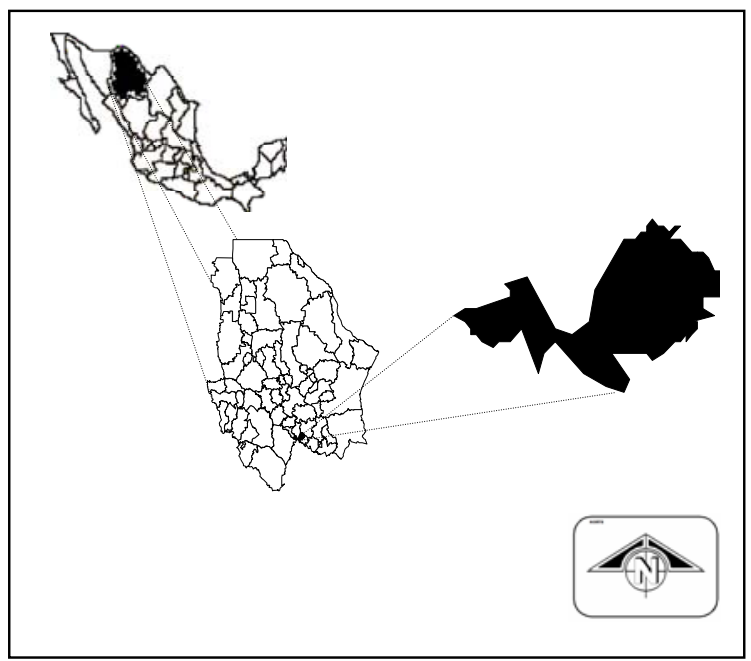

Figura 1. Localización del área de estudio en el Municipio San Francisco de Oro, Chihuahua.

Se tomaron muestras de suelo a diferentes niveles de profundidad: de $0-40,40-60$ y $60-80 \mathrm{~cm}$ en 10 sitios de muestreo, distribuidos sistemáticamente en el área de influencia de la presa de jales en dirección a los vientos dominantes, a una distancia de $300 \mathrm{~m}$ entre un punto y otro, es decir a los 300, 600, 900, 1200, $1500,1800,2100,2400,2700$ y 3000 m, para completar un total de 30 muestras.

Se determinaron las siguientes características físicoquímicas del suelo: textura, $\mathrm{pH}$, contenido de materia orgánica y conductividad eléctrica mediante la técnica propuesta por Uvalle-Bueno (1993) y modificada por Yáñez (1998).

Para el análisis de concentración de metales se preparó la muestra mezclando el suelo este se secó a temperatura ambiente durante 3 días, después se tamizó a $80 \mu \mathrm{m}$. Se tomaron $0.5 \mathrm{~g}$ de muestra y se colocó en un vial de teflón de microondas posteriormente se adicionó $4 \mathrm{~mL}$ de $\mathrm{HF}, 1 \mathrm{~mL}$ de HCL y $5 \mathrm{~mL}$ de $\mathrm{HNO}_{3}$ estos se pasaron en un digestor de microondas por 50 minutos a 120 psi y se agregó 2 g de $\mathrm{H}_{3} \mathrm{BO}_{3}$ en cristales. Después se analizaron mediante la técnica ICP (Inductively Coupled Plasma) las muestras con mayor contenido de elementos las que contenían menores concentraciones de contaminantes se leyeron mediante Absorción Atómico. Los metales pesados analizados fueron: As, $\mathrm{Pb}, \mathrm{Zn}$ y Cd.

El análisis estadístico consistió en generar modelos de regresión entre características físico-químicas, distancias, profundidades, para evaluar su relación con las concentraciones de metales pesados en suelo. El paquete estadístico utilizado fue Minitab. 


\section{Resultados}

El As en los sitios que se ubican a $300 \mathrm{~m}$ y $600 \mathrm{~m}$ de la presa de jales muestran altas concentraciones en las profundidades de 40-60 y 60-80 cm con 3670 y 5 410 ppm para el sitio 1 y de 3150 y 2930 ppm para el sitio 2. Esto indica que la concentración de este elemento ha pasado a estratos inferiores de suelo, debido a la gran cantidad de años que ha estado expuesto este a la contaminación. En el sitio 3 se presenta una alta concentración en la profundidad de 0-40 cm con $4265 \mathrm{ppm}$ pues este sitio se encuentra cercano a un arroyo que pasa en las cercanías de la presa de jales, por lo que el material es arrastrado y dejado en la superficie, sin tener oportunidad de lixiviarse por los escurrimientos del arroyo cuando hay precipitaciones importantes.

Las concentraciones totales de As se muestran en la Tabla 1, donde podemos observar que en la parte superficial las concentraciones de As sobre pasan las 1 000 ppm hasta el sitio 5 a $1500 \mathrm{~m}$ de distancia de los jales, dándose una disminución a medida que es mayor la distancia.

Tabla 1. Concentraciones de as (ppm) en suelo a diferentes profundidades y distancias de la presa de jales.

\begin{tabular}{cccccc}
\hline & \multicolumn{5}{c}{ DISTANCIA (m) } \\
$\begin{array}{c}\text { PROFUNDIDAD } \\
(\mathbf{c m})\end{array}$ & $\mathbf{3 0 0}$ & $\mathbf{6 0 0}$ & $\mathbf{9 0 0}$ & $\mathbf{1 2 0 0}$ & $\mathbf{1 5 0 0}$ \\
$\mathbf{0 - 4 0}$ & 2687 & 2588 & 4265 & 1566 & 1118 \\
$\mathbf{4 0 - 6 0}$ & 3670 & 3150 & 1120 & 340 & 490 \\
$\mathbf{6 0 - 8 0}$ & 5410 & 2930 & 310 & 330 & 470 \\
\hline \hline & \multicolumn{5}{c}{ DISTANCIA (m) } \\
PROFUNDIDAD & $\mathbf{1 8 0 0}$ & $\mathbf{2 1 0 0}$ & $\mathbf{2 4 0 0}$ & $\mathbf{2 7 0 0}$ & 3000 \\
$\mathbf{( c m )}$ & \multicolumn{5}{c}{} \\
\cline { 2 - 6 } $\mathbf{0 - 4 0}$ & 488 & 576 & 727 & 615 & 388 \\
$\mathbf{4 0 - 6 0}$ & 140 & 200 & 100 & 190 & 70 \\
$\mathbf{6 0 - 8 0}$ & 100 & 90 & 50 & 130 & 40 \\
\hline
\end{tabular}

Los valores que dan las diferentes agencias en relación al As en suelo, son muy variables la EPA (1992) maneja rangos entre 100 y 1000 ppm para suelo superficial, clasificándolos como sitios excesivamente fitotóxicos. En el área de estudio el rango de concentración en este estrato fue entre 552.66 y 4222.78 ppm, muy por encima de lo señalado, lo que da una clara idea del nivel tan alto de As que tienen estos suelos y el problema que representan a estos ecosistemas. Así mismo la agencia IHOBE (1998) señala 35 ppm de As como valor máximo de riesgo tolerable para protección de ecosistemas. Los resultados de este estudio muestran que todos los sitios y profundidades muestreados rebasan esta cantidad, algunos en forma muy significativa.

La USEPA (2001) señala como concentración máxima en suelo 100 ppm y recomienda realizar un proceso de remediación de sitio cuando se alcanza ese valor. En este estudio a excepción de 3 muestras en sitios lejanos a los jales y en profundidades no superficiales, todas las demás muestras rebasan esa cantidad. En México se dio a conocer el anteproyecto de NOM (2003) para limpieza y remediación de suelos contaminados dedicados a la agricultura, ahí se señalan 20 ppm como máximo permisible, lo que obligaría a la empresa que provocó esta contaminación a realizar una remediación de estos suelos, ya que varios de los sitios muestreados se les da ese uso.

El modelo de regresión para el As, muestra como variable predictora importante la distancia, que fue altamente significativa $(\mathrm{p}<0.000)$ con una tendencia negativa, ya que a medida que se alejan los sitios de la fuente de contaminación, esta disminuye. La variable distancia se asocia con la textura $(\mathrm{p}<0.060)$ y la materia orgánica del suelo $(\mathrm{p}<0.150)$, las tres con un valor de $\mathrm{R}^{2}=65.47$.

Tabla 2. Concentraciones de pb (ppm) en suelo a diferentes profundidades y distancias de la presa de jales.

\begin{tabular}{|c|c|c|c|c|c|}
\hline \multirow[b]{2}{*}{$\begin{array}{c}\text { PROFUNDIDAD } \\
\text { (cm) }\end{array}$} & \multicolumn{5}{|c|}{ DISTANCIAS (m) } \\
\hline & 300 & 600 & 900 & 1200 & 1500 \\
\hline $0-40$ & 1115 & 1142 & 2559 & 1115 & 1108 \\
\hline $40-60$ & 3000 & 1980 & 770 & 300 & 840 \\
\hline \multirow[t]{2}{*}{$60-80$} & 4720 & 1960 & 250 & 350 & 810 \\
\hline & \multicolumn{5}{|c|}{ DISTANCIAS (m) } \\
\hline $\begin{array}{c}\text { PROFUNDIDAD } \\
\text { (cm) }\end{array}$ & 1800 & 2100 & 2400 & 2700 & 3000 \\
\hline $0-40$ & 585 & 2122 & 823 & 967 & 706 \\
\hline $40-60$ & 240 & 270 & 150 & 190 & 170 \\
\hline $60-80$ & 130 & 180 & 100 & 160 & 110 \\
\hline
\end{tabular}

El $\mathrm{Pb}$ en los primeros 5 sitios ubicados dentro de los $1500 \mathrm{~m}$ de distancia a los jales, presentan valores arriba de las 1000 ppm en la parte superficial. En el sitio 1 se encontraron concentraciones elevadas de $\mathrm{Pb}$ en la profundidad de 60-80 cm con 4720 ppm seguida de la profundidad 40-60 cm con $3000 \mathrm{ppm}$. Se observa que el plomo en lugares cercanos a los jales tiene una concentración mayor a mas profundidad y en el resto de los sitios tiende a ir disminuyendo conforme se va alejando de la fuente de origen, a excepción del sitio 7 que presenta una concentración de 2122 ppm en la profundidad de 0-40 cm (Tabla 2), posiblemente por tratarse de un sitio que no contaba 
con vegetación arbustiva y era usado para la agricultura.

En relación a estos valores la EPA (1992) señala como rango entre 50 y 100 ppm para suelo superficial, clasificándolos como sitios excesivamente fitotóxicos. En el área de estudio el rango osciló entre 100 y 4720 ppm de $\mathrm{Pb}$ lo que indica un alto nivel de contaminación por este metal y un problema potencial para los organismos que viven en ese ecosistema. Por lo que respecta a la agencia IHOBE (1998) esta señala como valor máximo de riesgo tolerable para protección de ecosistemas 330 ppm. Todos los sitios en su parte superficial presentan niveles mayores a esta cantidad y solo los 6 sitios más distantes en las profundidades de 40-60 y 60-80 están por debajo de este valor, posiblemente por la distancia a la fuente de contaminación o porque el $\mathrm{Pb}$ no se lixivia fácilmente.

El anteproyecto de NOM (2003) para limpieza y remediación de suelos contaminados dedicados a la agricultura en México, da un valor máximo de 100 ppm. Con el rango obtenido en este estudio todos los sitios estarían por arriba de la normatividad, por lo que requerirían de medidas de remediación.

La USEPA (2001) recomienda hacer remediación del suelo en áreas de recreación infantil con un valor de 400 ppm y 1200 ppm de Pb en áreas de uso común. Tomando en cuenta que en esta zona del estudio se encuentra un centro escolar, los valores encontrados representan un peligro para la salud de los niños, e incluso las personas mayores.

El análisis de regresión para $\mathrm{Pb}$, muestra un nivel altamente significativo $(\mathrm{P}<0.002)$ con respecto a la distancia, también con una tendencia negativa, disminuyendo la concentración a medida que se aleja de la presa de jales, asociándose únicamente con la variable textura $(\mathrm{P}<0.088)$ aunque con un con valor bajo de $\mathrm{R}^{2}=43.72$ y un valor de alfa de 0.1 .

$\mathrm{El} \mathrm{Zn}$ al igual que el $\mathrm{Pb}$ y el As muestra en los dos primeros sitios las concentraciones mas elevadas, en el sitio 1 en la profundidad de 60-80 se eleva a 12050 ppm siendo este el valor mas alto. Esto coincide con Cabrera et al., (1999) que encontró en un estudio de un área minera concentraciones de Zn arriba de 11000 ppm. La profundidad de 40-60 presentó 7450 ppm. En el sitio 2 en la profundidad de 60-80 aparece con 3 490 ppm y en la de 40-60 3360 ppm en el sitio 3 es en la profundidad de 0-40 donde la concentración es alta con 3863 ppm. Los siguientes siete sitios presentan valores en la parte superficial de alrededor de 1000 ppm (Tabla 3) disminuyendo en los estratos inferiores, aunque con valores importantes. La EPA (1992), señala como sitios excesivamente fitotóxicos los que contienen entre 200 y 400 ppm de Zn. Los resultados encontrados muestran un rango entre $230 \mathrm{y}$ $12050 \mathrm{ppm}$, por lo que el nivel de contaminación debe considerarse como extremadamente alto. IHOBE (1998), señala como valor máximo de riesgo tolerable para protección de ecosistemas 840 ppm. En relación a este valor se puede observar que en la muestra superficial de suelo se rebasa este cantidad en todos los sitios, siendo menor únicamente en los sitios alejados a la fuente de contaminación en los estratos inferiores del suelo, por lo que si existe un problema de contaminación por este metal en la zona estudiada. Respecto al anteproyecto de NOM (2003) para limpieza y remediación de suelos contaminados dedicados a la agricultura en México, el valor máximo que propone es de $300 \mathrm{ppm}$, el cual es rebasado por casi todos los valores obtenidos, por lo que de aprobarse esta normatividad, el área de estudio requeriría de un proceso de remediación indiscutiblemente.

Tabla 3. Concentraciones de zn (ppm) en suelo a diferentes profundidades y distancias de la presa de jales.

\begin{tabular}{|c|c|c|c|c|c|}
\hline \multirow[b]{2}{*}{$\begin{array}{c}\text { PROFUNDIDAD } \\
\text { (cm) }\end{array}$} & \multicolumn{5}{|c|}{ DISTANCIAS (m) } \\
\hline & 300 & 600 & 900 & 1200 & 1500 \\
\hline $0-40$ & 1836 & 2818 & 3863 & 1787 & 1654 \\
\hline $40-60$ & 7450 & 3360 & 1060 & 540 & 1000 \\
\hline \multirow[t]{2}{*}{$60-80$} & 12050 & 3490 & 530 & 550 & 930 \\
\hline & \multicolumn{5}{|c|}{ DISTANCIAS (m) } \\
\hline $\begin{array}{c}\text { PROFUNDIDAD } \\
\text { (cm) }\end{array}$ & 1800 & 2100 & 2400 & 2700 & 3000 \\
\hline $0-40$ & 940 & 1294 & 1064 & 1295 & 1066 \\
\hline $40-60$ & 970 & 520 & 300 & 460 & 290 \\
\hline $60-80$ & 400 & 350 & 240 & 360 & 230 \\
\hline
\end{tabular}

El modelo de regresión muestra para el Zn un nivel altamente significativo $(\mathrm{P}<0.000)$ para la variable distancia comportándose de una forma similar al As y al $\mathrm{Pb}$, ya que a medida que se aleja de la fuente de contaminación disminuye la concentración. El Zn no se asocia de forma importante con ninguna de las variables estudiadas, mostrando sola un valor de $\mathrm{R}^{2}$ de 36.76 .

El Cd se comportó diferente a los otros metales ya que en todos los sitios mantuvo concentraciones más altas en la profundidad de $0-40 \mathrm{~cm}$ (Tabla 4). El valor más alto se encontró en el sitio 3 a 900 m de los jales, con una concentración de 204 ppm; este sitio corresponde a un punto cercano a un arroyo. En sitios a más de $2100 \mathrm{~m}$ de la fuente, en las profundidades de 40-60 y 60-80 cm no se detectaron concentraciones de este metal; así mismo se observó una clara disminución de la concentración en los niveles inferiores en relación al superficial, observándose una clara tendencia de este metal de permanecer en la parte superficial, lo que puede representar un mayor 
peligro para la población y la contaminación de aguas superficiales. Al igual que en los otros elementos estudiados se observa una clara tendencia a disminuir su presencia a medida que se aleja de la presa de jales.

Tabla 4. Concentraciones de cd (ppm) en suelo a diferentes profundidades y distancias de la presa de jales.

\begin{tabular}{|c|c|c|c|c|c|}
\hline \multirow[b]{2}{*}{$\begin{array}{c}\text { PROFUNDIDAD } \\
\text { (cm) }\end{array}$} & \multicolumn{5}{|c|}{ DISTANCIAS (m) } \\
\hline & 300 & 600 & 900 & 1200 & 1500 \\
\hline $0-40$ & 135 & 119 & 204 & 76 & 55 \\
\hline $40-60$ & 50 & 20 & 10 & 0 & 10 \\
\hline \multirow[t]{2}{*}{$60-80$} & 90 & 20 & 0 & 0 & 10 \\
\hline & \multicolumn{5}{|c|}{ DISTANCIAS (m) } \\
\hline $\begin{array}{l}\text { PROFUNDIDAD } \\
\text { (cm) }\end{array}$ & 1800 & 2100 & 2400 & 2700 & 3000 \\
\hline $0-40$ & 25 & 31 & 29 & 29 & 22 \\
\hline $40-60$ & 10 & 0 & 0 & 0 & 0 \\
\hline $60-80$ & 0 & 0 & 0 & 0 & 0 \\
\hline
\end{tabular}

La EPA (1992) contempla un rango de 3-10 ppm de $\mathrm{Cd}$ en suelo superficial, considerando sitios excesivamente fitotóxicos aquellos que lo rebasan. El promedio obtenido en este estudio a nivel superficial (0-40 cm) fue de 22 a 204 ppm de Cd, con una tendencia a disminuir a mayor distancia de los jales, pero siempre con valores mayores a los señalados. Considerando lo señalado por IHOBE (1998) que contempla $18 \mathrm{ppm}$ de Cd como valor máximo de riesgo tolerable para protección de ecosistemas y al del anteproyecto mexicano de norma NOM (2003) que es de 20 ppm, se observa que todos los sitios a nivel superficial rebasan este valor, por lo que el área de estudio presenta serios problemas de contaminación de suelo en relación al Cd.

El modelo de regresión muestra también una alta significancia $(\mathrm{P}<0.000)$ para la variable distancia, que se comportó similar a los otros elementos, con una tendencia negativa, asociándose con la variable profundidad de suelo en forma altamente significativa $(\mathrm{P}<0.000)$, también con una tendencia negativa, ya que el Cd tiende a no estar presente a medida que se aleja de la fuente de contaminación y en los estratos inferiores de suelo. La otra variable importante en el modelo fue el $\mathrm{pH}$ del suelo, con un nivel de significancia de $\mathrm{P}<0.114$, con tendencia negativa ya que a mayor profundidad del suelo el $\mathrm{pH}$ tiende a disminuir al igual que el $\mathrm{Cd}$. $\mathrm{La}$ variable conductividad eléctrica no resultó significativa en ninguno de los modelos de predicción de los elementos estudiados, teniendo un comportamiento sin una tendencia definida, lo que le dio una alta variabilidad y por lo tanto no intervino de forma significativa en los modelos de predicción.

\section{Discusión}

Los elementos As, $\mathrm{Pb}$ y $\mathrm{Zn}$ muestran un comportamiento similar, encontrándose en los 2 sitios más cercanos a la presa de jales concentraciones mas altas a mayores profundidades, lo que indica que estos elementos han pasado a esos estratos por la acumulación que desde hace cientos de años se ha dado en la zona de estudio. Esto coincide con Razo (2002) que encontró en profundidades entre 50 y 100 cm concentraciones de As mayores que en la parte superficial, en sitios cercanos a los jales. Para todos los elementos estudiados la variable distancia fue altamente significativa encontrándose una disminución de estos a medida que se aleja de la fuente de origen coincidiendo esto con Brimblecomebe (1994) y Roychowdhury et al. (2002) que señalan que existe un nivel de riesgo mayor de contaminación en las áreas cercanas a las presas de jales.

Los cuatro elementos muestran una tendencia a concentrarse mayormente en la capa superficial del suelo, a excepción de los dos primeros sitios, debido a que el depósito se da mayormente por efecto del viento, extendiéndose a distancias que pueden sobrepasar los $3 \mathrm{~km}$ de distancia de los jales. Esto coincide con Kabata-Pendias y Kabata- Pendias (1989), Simón et al. (1999) y Seoánez (1998) quienes mencionan que existe una tendencia de los metales pesados a acumularse en las capas superiores del suelo. Esta contaminación superficial ha provocado el abandono de tierras de cultivo en esta zona y en mucho de los casos la migración de los dueños de las mismas. En el caso de la siembra de algunos cultivos representa un riesgo para la salud por la posible acumulación de estos metales por las plantas.

Los metales pueden tardar de varias decenas a miles de años en reducir su volumen, ya que no pueden ser degradados; sólo se transforman a otros estados de oxidación en el suelo reduciendo su movilidad y toxicidad (McLean \& Bledsoe, 1992). No obstante, son un riesgo latente, ya que es en este estrato edáfico donde crecen las gramíneas y hierbas las cuales son consumidas por los animales y algunos de estos a su vez por el humano, entrando los metales a la cadena trófica. Al respecto Brus et al. (2002) señalan que las propiedades y características básicas del suelo, son las que determinan la transferencia de los metales pesados a las plantas.

Las variables predictivas para la concentración de As, mostraron a la textura y a la materia orgánica como variables importantes. Al respecto KabataPendias \& Pendias (2001), señalan que suelos con alto contenido de materia orgánica retienen el As 
adsorbiéndolo fuertemente y esto puede incrementarse a través de los años. En este estudio se encontró que en los sitios de mayor concentración de As, los contenidos de materia orgánica fueron muy bajos, no dándose una retención de este elemento y por consiguiente su lixiviación a los estratos inferiores del suelo. El pH no resultó significativo para este elemento, lo cual coincide con Williams (2001) Smedley \& Kinniburgh (2002), que señalan que las concentraciones de As no tienen una limitada movilidad en un rango amplio del pH del suelo.

El Pb también se asocia a la distancia a la fuente, así como a la textura del suelo. Al respecto Pérez et al. (1995) y Estévez et al. (1998), señalan que la textura tiene una función fundamental en la dinámica de los metales pesados en los suelos.

El Zn puede ser determinado en el modelo de regresión utilizando solo la variable distancia, aunque con un valor relativamente bajo de $r^{2}$. Esta variable tuvo el mismo comportamiento que el resto de los elementos; su presencia en cuanto a concentración es importante ya que de acuerdo a Razo (2002) el Zn es considerado el elemento más soluble en suelos alcalinos con respecto al resto de los metales pesados, teniendo esta característica el $60 \%$ de los sitios y estratos muestreados.

El Cd, metal que en el modelo de regresión mostró asociación importante con la distancia al igual que el resto de los elementos, es considerado peligroso en bajas concentraciones. Otras variables importantes de predicción fueron la profundidad del suelo y el $\mathrm{pH}$ ambas en forma negativa lo cual coincide con lo citado por Anderson \& Christensen (1988) encontraron en un estudio que el $\mathrm{pH}$ fue el factor que más influyó en controlar la distribución del Cd en suelo. Por lo que respecta a su movilidad se encontró que este metal tiende a permanecer mayormente en la superficie, siendo esta la causa que la variable profundidad de suelo resultó importante.

El impacto sobre el recurso suelo por efecto de las altas concentraciones de los elementos estudiados ha generado una serie de problemas en el área de estudio entre los que se encuentran muerte de animales, enfermedades crónico degenerativas en las personas, baja o nula productividad agrícola, así como enfrentamientos entre la compañía minera y los pobladores.

\section{Conclusiones}

Los cuatro elementos estudiados, presentaron una mayor acumulación en los 2 sitios cercanos a la presa de jales, esto es los primeros $600 \mathrm{~m}$. En estos dos sitios la concentración de $\mathrm{As}, \mathrm{Pb}$ y $\mathrm{Zn}$, fueron mayores en las profundidades de 40-60 y 60-80 cm que en la superficie.

El Cd fue el único metal que no presentó esta característica y que en estos sitios presentó mayor concentración en la superficie.
Las concentraciones de los elementos estudiados sobrepasan los rangos propuestos por diferentes agencias internacionales, por lo que es urgente una remediación de esta área.

La distancia, fue la variable más importante en los modelos de predicción.

El Zn se encontró en cantidades muy altas en toda el área, por lo que debe considerarse como riesgoso, para las plantas.

El Cd se correlacionó con el $\mathrm{pH}$, con la distancia en forma negativa, al igual que con la profundidad de suelo.

En base a los resultados obtenidos, se concluye que estos suelos deberán ser sometidos a un proceso de remediación, con la finalidad de incorporarlos a los procesos productivos sin riesgo para los componentes de este ecosistema.

\section{Literatura Citada}

Anderson P.R. \& Christensen T.H. 1988. Distribution coefficients of Cd, Co, Ni and Zn in soil. J. Soil Sci. 39: 15-22.

Anteproyecto de Norma Oficial Mexicana. 2003. Que establece criterios para determinar niveles de limpieza para la remediación de suelos contaminados por metales y metaloides. Secretaría del Medio Ambiente y Recursos Naturales.

Baird C. 1999. Environmental Chemistry. $2^{\text {nd }}$ Ed. W.H. Freman \& Company.

Brimblecomebe P. 1994. Cambios a largo plazo en la depositación elemental de la superficie de la tierra. Contaminación ambiental. 83: 81-85.

Brus D.J. de Gruijter, Walvoort D.J.J., de Vries F., Bronswijk J.J.B., Romkens P.F.A.M. \& de Vries W .2002. Mapping the probability of exceeding critical thresholds for cadmium concentrations in soils in the Netherlands. J. Environ. Qual. 31: 1875-1884.

Cabrera F., Clemente L., Díaz B.E., López R. \& Murillo J.M. 1999. Heavy metals pollution of soil affected by the Guadiamar toxic flood. Sci. Total Environ. 2242: 117-129.

Colombo L.D., Mangione S.B. \& Figlioglia A. 1998. Soil profile distribution of heavy metals in soil atended with sewage sludge for eight years. Agr. Med. Inter. J. of Agric. Sci. 128: 273-283.

EPA. 1992. Guide to site and soil description for hazardous waste site characterization. Vol 1: metals.

Estévez J.I., Andrade L., Marcel P. \& Montero J.M. 1998. La influencia de las propiedades del suelo en la fijación y movilidad de Zn y Cd. Edafología: Sociedad Española de la ciencia del suelo. 5: 19-27.

García I. \& Dorronsoro C. 2002. Contaminación por metales pesados. Departamento de edafología y química agrícola de España.

Gulson B.L., Mizon K.J., Korsch M.J. \& Howarth D. 1996. Non-orebody sources are significant contributors to blood lead of some children with low to moderate lead exposure in a mayor mining community. The science of the total environment. 181: 223-230.

IHOBE (Seguridad pública de gestión ambiental). 1998. Manual práctico para la investigación de la 
contaminación del suelo. Seguridad pública de gestión ambiental. Gobierno Vasco, España.

Kabata - Pendias A. \& Kabata - Pendias Kh. 1989. Macroelements in the soil and plants. Myr., Moskva (in Russian).

Kabata-Pendias A. \& Pendias H. 2001. Trace elements in soils and plants CRC. Press, Florida.

McLean E.J. \& Bledsoe B.E. 1992. Behavior of metals. Ground water issue. EPA/540/S-92/018.

Pérez C.L., Moreno A. M. \& González J.P. 1995. Influencia de la fracción arcilla y arena en el contenido y disponibilidad de metales pesados en el suelo. Edafología: Sociedad Española de la ciencia del suelo. 1: 83-89.

Razo S.I. 2002. Evaluación de la contaminación por metales y del riesgo en salud en un sitio minero de sulfuros polimetálicos: caso de Villa de la Paz-Matehuala S.L.P (México). Tesis maestría. Instituto de metalurgia, Facultad de Ingeniería, Universidad Autónoma de San Luis Potosí.

Roychowdhury T., Uchino T., Tokunaga H. \& Ando M . 2002. Arsenic and other heavy metals in soil from an arsenic-affected area of West Bengal, India. Chemosphere. 6: 605-618.

Salomon W. 1995. Environmental impact of metals derived from mining activities: processes, predictions, prevention. J. Geochim. Explor. 52: 5-23.
Seoánez C.M. 1998. Contaminación de suelos: estudios, tratamiento y gestión. Ediciones mundi prensa.

Simón M. Ortiz I., García I., Fernández E., Fernández J., Dorronsoro C. \& Aguilar J. 1999. Pollution of soil by the toxic spill of a pyrite mine (Aznalcóllar, Spain). Sci Total Environ. 242: 105-115.

Smedley P.L. \& Kinniburgh D.G. 2002. A review of the source, behaviour and distribution of arsenic in natural waters; Applied Geochenistry.17: 517-568.

USEPA (United State Environmental Agency). 2001. Residential lead hazard standards-TSCA section 403: office of pollution prevention and toxics. Environmental protection Agency, U.S.

Uvalle-Bueno J.X. 1993. Identificación de las propiedades del suelo asociadas con la clorosis en soya. Agricultura Técnica en México. Año 39. 1.19.(1).

Williams M. 2001. Arsenic in mine water; An International Study. Environmental Geology. 40: 267-278.

Wong M.H. 2003. Ecological restoration of mine degraded soils, with emphasis on metal contaminated soils. Chemosphere. 6: 775-780.

Yáñez R.M. 1998. Generación de estándares nutricionales y caracterización nutrimental preliminar del nogal pecadero "Western Schley" mediante el diagnostico diferencial integrado. Tesis de maestría. Facultad de Ciencias Agrotecnológicas, UACh.

${ }^{1}$ Estudiantes de doctorado, ${ }^{2}$ Maestro Investigador, Facultad de Zootecnia, Universidad Autónoma de Chihuahua. Periférico Francisco R. Almada Km.1. Email: spuga2002@yahoo.com.mx. 